\title{
Development of contractile properties in the fetal porcine urinary bladder
}

\author{
Lotte K Jakobsen $^{1,4}$, Karina F Trelborg ${ }^{1,4}$, Ulf Simonsen ${ }^{2}$, Karl-Erik Andersson ${ }^{1}$ and L H Olsen ${ }^{1,3}$
}

BACKGROUND: In early fetal life, the bladder is merely a conduit allowing urine to pass through freely into the amniotic cavity. As the striated external urethral sphincter evolves, the bladder acquires its reservoir and voiding functions. We characterized the myogenic and neurogenic contractions of the normal fetal porcine bladder from midterm until close to full-term gestation.

METHODS: Contractile responses were measured in vitro using bladder strips from fetuses at $60(N=23)$ and 100 days $(N=21)$ of gestation. Spontaneous activity, and the responses to potassium chloride $(\mathrm{KCl})$ solution, electrical field stimulation (EFS), and receptor activation were recorded. The smooth muscle content was evaluated histologically.

RESULTS: Histological studies revealed that the fractional content of smooth muscle doubled between the two time points, and passive tension was adjusted to take that into account. Spontaneous activity was regular at 60 days, changing toward an irregular pattern at 100 days. Contractile force elicited by $\mathrm{KCl}$ and carbachol increased significantly with gestational age, while contractions to the purinergic agonist, a- $\beta$-methylene adenosine 5'-triphosphate did not. The responses to EFS were almost completely blocked by atropine. CONCLUSION: Spontaneous myogenic contractions become irregular and contractile responses to muscarinic receptor stimulation increase during gestation, as the bladder reservoir and voiding functions develop.

$T^{1}$ he bladder develops from the cranial part of the urogenital sinus. Cells of mesenchymal origin differentiate into fibroblasts and smooth muscle cells, forming the detrusor layer. Endodermal cells differentiate into the urothelium. A milestone in the development of the human bladder is the formation of the external striated urethral sphincter during week 10 of gestation. At this time, smooth muscle cells can also be detected in the bladder wall. After this point, the bladder gradually changes from a conduit into displaying reservoir capabilities, and micro-motions are observed in the emerging detrusor (1-4).
Functional studies of the fetal sheep and calf bladders show that the detrusor muscle is fully developed halfway to three quarters through gestation, but with the functional innervation continuing to increase toward the term $(5,6)$. This time course of development is concordant with cystometries performed in utero on fetal pigs at 62 and 80 days of gestation thought to correspond to the mid-second trimester and early third trimester in human pregnancy. These investigations showed the development of storage capability and active voiding within this time frame. At mid-second trimester, the bladder served as a conduit, whereas mature types of voiding were recorded in the older gestation group $(2,7)$. Structural studies in human fetuses show the appearance of a smooth muscle in the bladder wall as early as gestational weeks 7-11, but with an increase in thickness throughout gestation (8). In addition, a positive reaction for tissue acetylcholinesterase has been demonstrated in the bladder smooth muscle from the 12th gestational week and onwards (8).

The similarities in the anatomy and physiology, demonstrated in several studies on adult animals, suggest that the pig is a good model for studying human conditions in the urinary tract. Comparable urodynamic characteristics and the ability to urinate spontaneously at birth further support this suggestion (9-11). However, most studies addressing fetal bladder development have been conducted on other animals. The unpredictable nature of congenital malformations, such as posterior urethral valves, poses a major challenge when planning treatment. Knowledge of the normal time course during fetal development is essential to understand and manage the pathological consequences of such congenital malformations. Many of these malformations manifest prenatally, and early intervention may be an option to reduce the damage and preserve renal and bladder function. It would be of great value to have a porcine model of congenital urogenital malformations, to test the results of intrauterine treatment and postnatal management, since determining the optimal timing for intervention is challenging. To provide a basis for making such determinations, we have characterized the development of myogenic and neurogenic contractions of the normal fetal porcine bladder from midterm until close to full term.

\footnotetext{
${ }^{1}$ Department of Clinical Medicine, Aarhus University, Aarhus, Denmark; ${ }^{2}$ Department of Biomedicine, Aarhus University, Aarhus, Denmark; ${ }^{3}$ Department of Urology, Aarhus University Hospital, Aarhus, Denmark. Correspondence: Lotte K. Jakobsen (LotteKJ@clin.au.dk)

${ }^{4}$ These authors contributed equally to the work.

Received 29 December 2016; revised 3 May 2017; accepted 18 July 2017; advance online publication 4 October 2017. doi:10.1038/pr.2017.181
} 


\section{Contractile properties in fetal bladder $\quad$ Articles}

\section{METHODS}

\section{Experimental Animals}

Pregnant sows (crossbred Danish Landrace/Yorkshire, 270-305 kg, inseminated with Duroc semen) were killed by intravenous injection of pentobarbital at 60 or 100 days of gestation (the average gestation length in these pigs is 114 days). Ethical approval of the experiments was granted by the Danish Animal Experiments Inspectorate (Approval no. 2014-15-0201-00356). All procedures were carried out at Aarhus University and conformed to the Danish National Guidelines for care and handling of animals, and to the published guidelines from the National Institute of Health (12). The uterus was excised and opened, and the fetuses were weighed and necropsied (60 days: $N=23,100$ days: $N=21$ ). The majority of the fetuses were female ( 60 days: female $=13$, male $=8$, and unknown $=2,100$ days: female $=15$, male $=6$ ), and the median (interquartile range (IQR)) weight of the fetuses was $116 \mathrm{~g}(108-124 \mathrm{~g})$ in the 60-day group vs. $801 \mathrm{~g}(744-859 \mathrm{~g})$ in the 100 -day group. The fetal bladders were excised and stored in ice-cold, oxygenated physiological salt solution (PSS) buffer until preparation for in vitro studies, or placed in $10 \%$ formalin for histological examination.

\section{Tissue Handling and Preparation for In Vitro Studies}

Bladders were opened by a midline sagittal incision, and pinned out in a Petri dish covered by PSS (1.19 mM NaCl, $4.7 \mathrm{~mm} \mathrm{KCl,} 1.17 \mathrm{~mm}$ $\mathrm{MgSO}_{4} \bullet 7 \mathrm{H}_{2} \mathrm{O}, 25 \mathrm{~mm} \mathrm{NaHCO}, 1.18 \mathrm{mM} \mathrm{KH} \mathrm{KH}_{2}, 1.61 \mathrm{CaCl}_{2}$, $0.026 \mathrm{~mm}$ EDTA, and $5.5 \mathrm{~mm}$ glucose). From each bladder, two to five longitudinal, whole-wall bladder strips $(1 \mathrm{~mm}$ wide and $3 \mathrm{~mm}$ long) were dissected from the mid-portion of the bladder. The strips were tied with 6-0 silk suture loops at both ends, and mounted in organ bath chambers with one end attached to a fixed steel pin, and the other end attached to an isometric force transducer. Throughout the experiment, the chambers were filled with PSS at $37^{\circ} \mathrm{C}$ at a pH of 7.4, continuously aerated with $95 \% \mathrm{O}_{2}$ and $5 \% \mathrm{CO}_{2}$. PSS was changed at least every $30 \mathrm{~min}$. The strips that were not used immediately for experiments were stored in ice-cold, oxygenated PSS. Three organ bath 4-chamber settings from Danish Myo Technology (Aarhus, Denmark) were used, enabling simultaneous experiments on 12 strips (model 750TOBS for electrical field stimulation (EFS) experiments, two $700 \mathrm{MO}$ models for all other experiments). The chambers contained a volume of either $5 \mathrm{ml}$ (horizontal chambers) or $10 \mathrm{ml}$ (vertical chambers). Isometric contractile responses were sampled using a Powerlab (ADInstruments, Dunedin, New Zealand), and the data were recorded in LabChart v.7.0 (ADInstruments). At the end of each experiment, suture loops were cut off, and the strips were weighed (strip weight, median (IQR): 60 days: $3.66 \mathrm{mg}$ (3.23-4.08), 100 days: $13.26 \mathrm{mg}$ $(11.64-14.89 \mathrm{mg}))$. Each strip was used in either force-tension experiments, pharmacological experiments (including spontaneous activity recordings and $\mathrm{KCl}$ activation) or EFS experiments (including $\mathrm{KCl}$ activation). A total of $n=59$ strips from the 60 -day group and $n=60$ strips from the 100-day group were used.

\section{Determination of the Optimal Basal Tension}

To obtain information about the optimal basal tension for each gestational age, the study was started with force-tension experiments $(N=8$ in each group). Following mounting and a 20 -min equilibration phase, the tissue preparations were stretched in $1-\mathrm{mN}$ increments. Starting at $4 \mathrm{mN}$ for 60 days and $5 \mathrm{mN}$ for 100 days, the tissue was stimulated with $\mathrm{KCl}(123.7 \mathrm{~mm} \mathrm{KCl}, 1.17 \mathrm{~mm}$ $\mathrm{MgSO}_{4} \bullet 7 \mathrm{H}_{2} \mathrm{O}, 25 \mathrm{~mm} \mathrm{NaHCO}_{3}, 1.18 \mathrm{~mm} \mathrm{KH}_{2} \mathrm{PO}_{4}, 1.61 \mathrm{CaCl}_{2}$, $0.027 \mathrm{~mm}$ EDTA, and $10 \mathrm{~mm}$ glucose) following each tension increment. Tension was increased until the difference in force generation between two stimulations was $<10 \%$. The tension producing just below the maximal response (the second last) was considered the optimal basal tension and was applied throughout the remaining experiments. The strips used in force-tension experiments were weighed and discarded, and fresh strips were used for the remaining experiments.
Spontaneous Activity Recordings

Following the force-tension experiment, fresh tissue preparations were mounted, equilibrated, and stretched to the optimal basal tension $(N=12, n=16$ in each group). Spontaneous activity was then recorded for a period of $30 \mathrm{~min}$ before the first $\mathrm{KCl}$ activation and again for $30 \mathrm{~min}$ before the second $\mathrm{KCl}$ activation. The frequency and amplitude of spontaneous contractions were calculated from a representative 5 -min period from each preparation. The frequency of contractions was calculated, considering all positive peaks crossing the baseline. The amplitude represents the difference between baseline tension and peak tension in each of these contractions. The baseline is represented by the basal tension of $6 \mathrm{mN}$ in 60 -day preparations and $8 \mathrm{mN}$ in 100-day preparations. However, in some strips, too many peaks of contractions would be ignored by calculations based on this baseline tension and, in these cases, a fitted baseline was applied. Similarly, the area under the curve was calculated as the total area between baseline tension and the curve of spontaneous activity, not considering the peaks below the baseline. The frequency components of spontaneous activity were further quantified by spectral analysis, using a fast Fourier transformation (FFT) to decompose the signal into various frequencies. FFT of the recorded signal was performed in Matlab version 2016a (MathWorks, Natick, MA), and the median frequency is reported with IQR. While the manual calculations were performed on representative 5-min periods, the FFT was performed on 15-min periods of spontaneous activity recordings.

\section{Potassium Chloride Activation}

Before either pharmacological or electrical stimulation, after the first recording of spontaneous activity, the chambers were filled with $125 \mathrm{~mm} \mathrm{KCl}$ instead of PSS. When a plateau of maximum contraction was reached, preparations and chambers were washed and filled with PSS again. This $\mathrm{KCl}$ activation was repeated, and if the difference in contractile response was more than $10 \%$ between the two, then, a third $\mathrm{KCl}$ activation was performed. To ensure that the contractile capability of the tissue was preserved throughout the duration of the experiments, activation with $125 \mathrm{mM} \mathrm{KCl}$ was repeated at the end of the experiments. The mean amplitude of potassium-induced contractions was calculated, and the responses were normalized to the strip weights ( $n=48$ in each group).

\section{Pharmacological Stimulation}

Strips for pharmacological stimulation were mounted in $5 \mathrm{ml}$ horizontal chambers. They were exposed to three single concentrations $\left(10^{-7}, 10^{-6}\right.$, and $\left.10^{-5} \mathrm{M}\right)$ of $\alpha-\beta$-methylene adenosine $5^{\prime}-$ triphosphate (ABMA) (Sigma-Aldrich, St Louis, MO), where $N=12$ and $n=16$ in each group. A careful washout was performed after each application. Next, an eight-step cumulative concentrationresponse curve to carbachol $\left(10^{-7}\right.$ to $10^{-4} \mathrm{M}$ ) (Sigma-Aldrich) was performed, with each dose added when the previous response reached a plateau (60 days: $N=23, n=28,100$ days: $N=21, n=27$ ). The distance from the baseline to the plateau level of each concentration was calculated and normalized to the strip weight. The carbachol concentration needed to produce a half maximal contraction $\left(\mathrm{EC}_{50}\right)$ was calculated in GraphPad Prism 7.0a (GraphPad Software, La Jolla, CA) using a nonlinear curve-fit model:

$$
y=\text { bottom }+\frac{(\text { top }- \text { bottom })}{1+10^{\left(\left(\log \mathrm{EC}_{50}-x\right) * \text { slope }\right)}}
$$

with constraints on slope (1) and bottom (0), since the responses were measured from the baseline.

\section{Electrical Field Stimulation}

Strips for EFS (60 days: $N=7, n=7,100$ days: $N=6, n=6$ ) were mounted in $10 \mathrm{ml}$ vertical chambers, between two parallel platinum electrodes, connected to an S88 stimulator (Grass Instruments, Rhode Island, NY). Following the initial $\mathrm{KCl}$ activation, the strips were stimulated with electrical pulses (pulse duration $0.5 \mathrm{~ms}$, train duration $5 \mathrm{~s}$, train delay $120 \mathrm{~s}$ and current $80-100 \mathrm{~mA}$ ) in six steps of 


\section{Articles $\mid$ Jakbsen et al.}

a

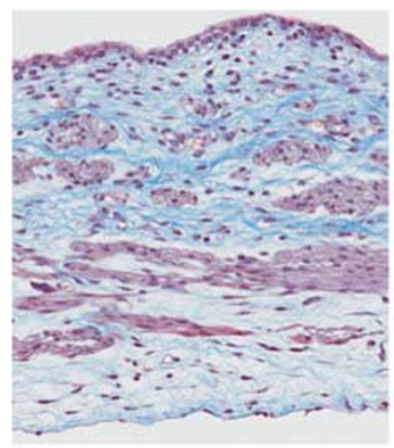

$100 \mu \mathrm{m}$ b
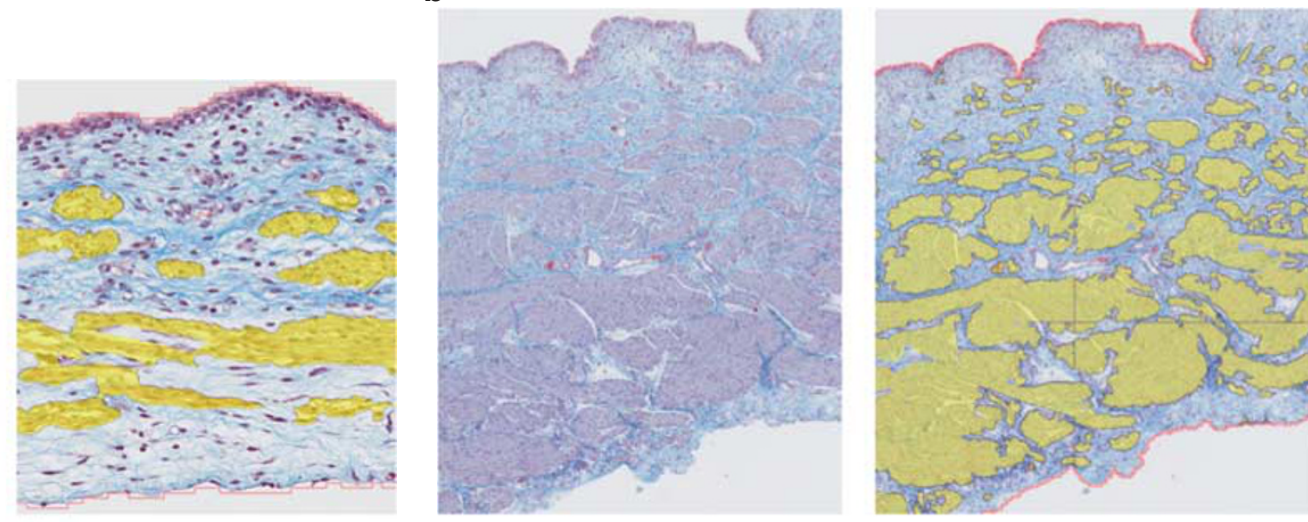

$400 \mu \mathrm{m}$

Figure 1. Histological images. Sections of fetal porcine bladder at 60 (a) and 100 (b) days of gestation, stained with Masson's Trichrome. The same sections are shown adjacent to each other with and without running the image analysis APP. The yellow areas indicate the area counted as the muscle.

increasing frequency starting at $2 \mathrm{~Hz}$, and doubling until $64 \mathrm{~Hz}$. After the first frequency-response cycle, atropine $\left(10^{-6} \mathrm{M}\right)$ (SigmaAldrich) was added and the electrical stimulation was repeated, then ABMA $\left(10^{-5} \mathrm{M}\right)$ was added and another cycle of EFS was performed. There was no washout between atropine and the addition of ABMA. Finally, after washout, tetrodotoxin (TTX: $10^{-6} \mathrm{M}$ ) (BioNordika, Herlev, Denmark) was added before a final frequencyresponse cycle to test whether the responses were mediated through the stimulation of nerves.

\section{Histology}

Fetal porcine bladders were fixed in formalin for $24 \mathrm{~h}$ and subsequently dehydrated with increasing ethanol solutions, placed in xylene, and embedded in paraffin. Sections $(3 \mu \mathrm{m})$ were cut, deparaffinized, rehydrated, and stained with Masson's Trichrome. Slides were scanned in Nanozoomer 2.0-HT $(\times 20)$ (Hamamatsu Photonics, Iwata City, Japan), and image analysis was performed in Visiopharm Software version 6.1.0.1895 (Visiopharm, Hørsholm, Denmark). From the 60-day group, six bladders (female $=3$, male $=3$ ) and from the 100-day group, seven bladders (female $=3$, male $=4$ ) were included. Two sections from each bladder were evaluated. Regions of interest (ROIs) were traced manually, including all sections or parts of sections, bordered on one side by urothelium and on the other side by serosa, thus ensuring the inclusion of the entire bladder wall. An application protocol package (APP) was created at $\times 10$ magnification to distinguish between the areas of redstained muscular tissue and other tissues within the ROI. Red-green contrast was chosen as the best feature for this. Unsupervised K-means classification using five classes was performed, which after cleanup, identified the muscle tissue with a yellow label. The cleanup consisted of removing red-stained vessels, nuclei, and urothelium while closing artifact holes from tissue processing. After a minor manual cleanup, an APP was deployed, calculating the area fraction of a yellow label (muscle) to the total tissue area of the ROI (Figure 1).

\section{Statistical Analysis}

GraphPad Prism 7.0a (GraphPad Software) was used for statistical analyses. The curves of carbachol concentration-response relation were compared between groups by two-way repeated measures ANOVA, followed by Sidak's multiple comparisons test. Group means of individual responses to $\mathrm{KCl}, \mathrm{ABMA}$, and $\mathrm{EFS}$, as well as the carbachol $\mathrm{EC}_{50} \mathrm{~s}$ and fractional smooth muscle areas were compared by unpaired $t$-tests. The responses to $\mathrm{KCl}$ activation before and after the experiments were compared by a paired $t$-test. The results were considered statistically significant when $P<0.05$. The results are reported as mean and $95 \%$ confidence intervals, unless otherwise stated. QQ plots were constructed to test the adherence to Gaussian distribution. In cases of unequal variances, compared by the F-test, Welch's correction was applied. Numerical data on spontaneous activity (frequency, amplitude, and AUC) did not adhere to the Gaussian distribution, and were therefore compared between groups by Mann-Whitney $U$-test, and within groups by Wilcoxonmatched pairs signed-rank test. These results are reported as median and IQR.

\section{RESULTS}

Four pregnant sows were killed. We included 23 bladders from fetuses at 60 days of gestation and 21 bladders from fetuses at 100 days of gestation for the organ bath experiments. For histology, we included six bladders from the 60-day group and seven bladders from the 100-day group.

\section{Histology}

The images are shown in Figure 1. The bladder wall structure was similar in both groups. Bundles of the detrusor muscle are separated by a connective tissue, and covered on the luminal side by the lamina propria and the multilayered urothelium. However, the mean fractional area of the smooth muscle in the bladder wall at 60 days of gestation was only $18.5 \%$ (CI: 16.5-20.5\%), which was significantly lower than $40.5 \%$ (CI: $38-43 \%)$ found at 100 days $(P<0.01)$.

\section{Force-Tension Experiments}

In both groups, an increase in generated force was observed when increasing basal tension. The basal tension producing just below the maximum force was $6 \mathrm{mN}$ for 60 days and $8 \mathrm{mN}$ for 100 days of gestation. These basal tensions are considered the optimal tensions for stimulation, and were thus maintained throughout the experiments. 


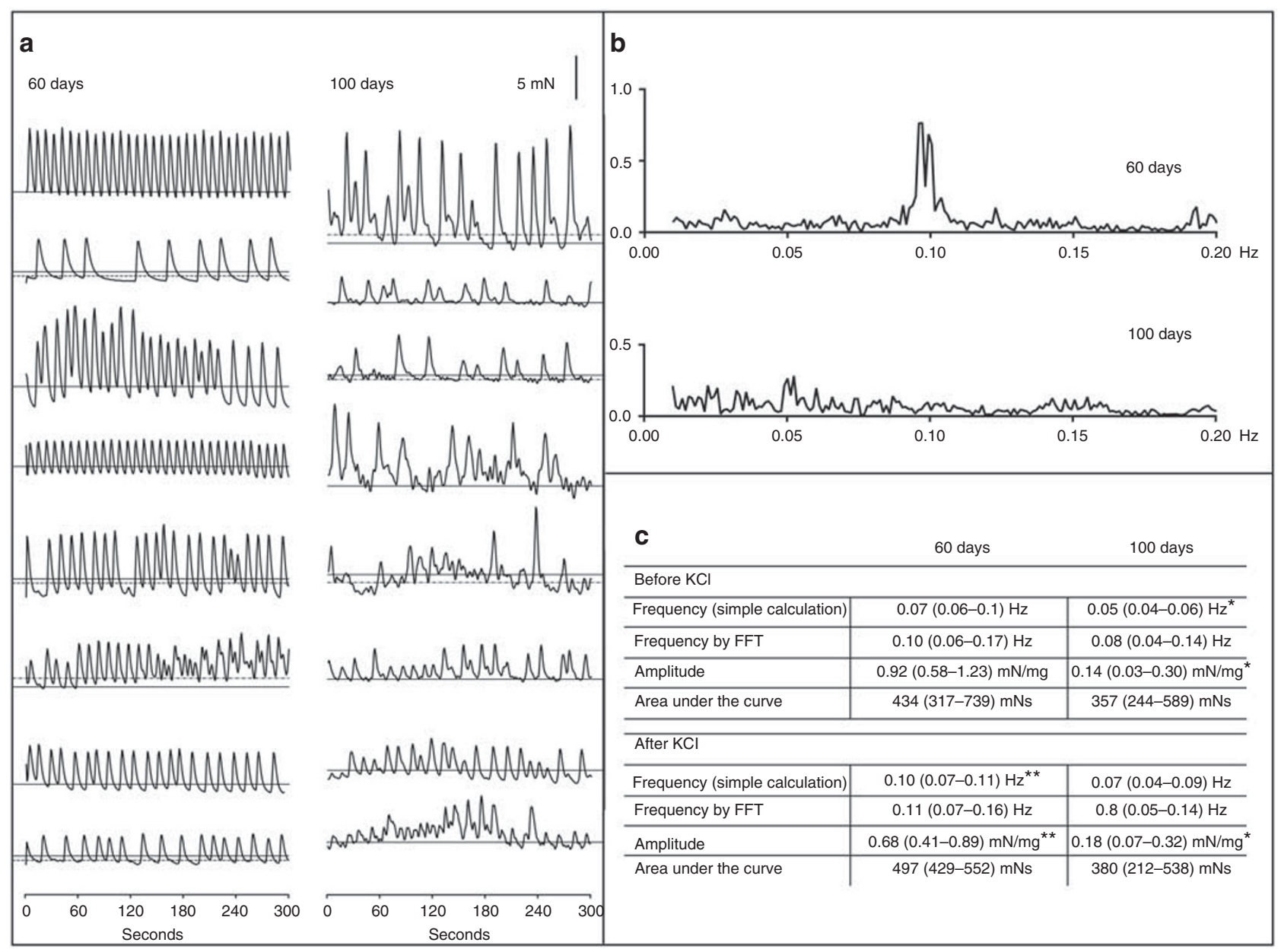

Figure 2. Spontaneous activity. (a) Five-minute representative traces of spontaneous activity before $\mathrm{KCl}$ activation from eight strips in each group. The solid line indicates the basal tension of $6 \mathrm{mN}$ for the 60-day group and $8 \mathrm{mN}$ for the 100-day group. Since the activity fluctuates a fitted baseline is applied in some cases, as indicated by an additional dotted line in the relevant traces. The activity pattern differs between the strips, but the overall impression of a regular pattern at 60 days and an irregular pattern at 100 days is evident. (b) Examples of fast Fourier transformations (FFTs) on $15 \mathrm{~min}$ of spontaneous activity. There is a frequency peak in most 60-day strips close to $0.1 \mathrm{~Hz}$, whereas the frequency spectrum in most 100 -day strips is more flat. The $Y$-axis indicates the relative contribution from each frequency component. (c) Table with numerical data. Frequency is calculated from the number of peaks crossing the baseline (a) during the 5-min period (simple), or by the FFT. The amplitude of each contraction is calculated as the difference between the baseline and the peak force and normalized to the strip weight. AUC is calculated as the total area between the baseline and trace during the 5 -min period. All are reported as median and IQR. *Significant difference between the groups (60 days vs. 100 days), $P<0.05$. * Significant difference within the groups comparing the results before and after $\mathrm{KCl}$ activation, $P<0.05$.

\section{Muscle Depolarization with Potassium Chloride}

Force generation caused by muscle depolarization with $125 \mathrm{mM} \mathrm{KCl}$ was significantly higher at 100 days $(6.0 \mathrm{mN} /$ $\mathrm{mg}, 5.2-6.7 \mathrm{mN} / \mathrm{mg})$ than at 60 days $(3.3 \mathrm{mN} / \mathrm{mg}, 2.9-$ $3.7 \mathrm{mN} / \mathrm{mg}$ ). The final responses were not significantly different from the initial responses, indicating that the viability of the tissue was maintained throughout the duration of the experiment.

\section{Spontaneous Activity}

All preparations exhibited spontaneous contractions. Representative 5-min tracings are shown in Figure 2a, along with a table of the numerical data (Figure 2c) and FFT results (Figures $\mathbf{2 b , c )}$. There is a wide variety of patterns between the strips as well as pattern variations over time in most strips.
However, the overall pattern of spontaneous activity was clearly different between the groups, with a pattern of regular homogeneous contractions at 60 days of gestation, changing toward an irregular pattern of non-homogeneous contractions at 100 days of gestation. Both the frequency and amplitude of contractions were higher in the 60-day group, even though these data are not normalized to the strip weight. The area under the curve is only slightly higher in the 60-day group, due to the broader composite contractions observed in the 100-day group. The pattern did not change significantly after $\mathrm{KCl}$ activation in the 100-day group. Regarding the 60-day group, the pattern after $\mathrm{KCl}$ activation was even more homogeneous than before, with an increased frequency and decreased amplitude of contractions (curves after $\mathrm{KCl}$ not shown). Since we were only able to record the activity in eight 


\section{Articles $\mid$ Jakobsen et al.}

strips at a time, half of the strips were stored for several hours before recording. This did not markedly affect the activity in the 60-day group, whereas the majority of stored strips from the 100-day group showed less spontaneous activity. Numerical data are therefore based solely on recordings from fresh strips. The patterns of activity were similar between fresh and stored strips. The FFT showed a wider frequency spectrum in the 100-day group. A frequency peak close to $0.1 \mathrm{~Hz}$ in the FFT occurred in the majority of the 60-day strips, whereas most strips in the 100-day group showed no obvious peaks (Figure 2b). Merging FFT data from all strips indicated a higher median frequency in the 60-day group than in the 100day group. This difference is more pronounced after depolarization with $\mathrm{KCl}$, which shifted the frequency spectrums of both groups toward higher frequencies (Figure 2c).

\section{Responses to Purinergic Receptor Stimulation by ABMA}

The results are shown in Figure 3. There was no response to the low concentration $\left(10^{-7} \mathrm{M}\right)$ of ABMA in either group. Stimulation with $10^{-6}$ and $10^{-5} \mathrm{M}$ caused responses in both groups. There was a tendency toward a greater response in the 60 -day group when applying the $10^{-6} \mathrm{M}$ dose, but this difference was not significant $(P=0.14)$. After application of the higher concentration, there was no significant difference between the groups $(P=0.38)$, but there was a wide variability of force generated, following a high-dose administration in the 100-day group.

\section{Responses to Muscarinic Receptor Stimulation by Carbachol} Concentration-response curves to carbachol are shown in Figure 3. All strips contracted in response to all carbachol concentrations, except for six strips (21\%) in the 60-day group and four strips (15\%) in the 100-day group that did not respond to the lowest carbachol concentration $\left(10^{-7} \mathrm{M}\right)$. The lowest concentration elicited a significantly more powerful response in the 60-day group than in the 100-day group $(P<0.01)$, but, overall, there was a significantly greater response from the 100 -day group $(P<0.01)$. The mean $\mathrm{EC}_{50}$ was also significantly higher in the 100-day group, $8.7 \times 10^{-7} \mathrm{M}$ (CI: $\left.6.4 \times 10^{-7}-10.9 \times 10^{-7} \mathrm{M}\right)$ compared to the 60 -day group, $1.9 \times 10^{-7} \mathrm{M} \quad\left(\mathrm{CI}: \quad 1.3 \times 10^{-7}\right.$ $\left.2.4 \times 10^{-7} \mathrm{M}\right)$ where $P<0.01$.

\section{Responses to Electrical Field Stimulation}

The results from EFS are shown in Figure 4. Six strips in the 100 -day and seven strips in the 60-day group were included. The contractile force was not significantly different between the two groups, but there was a tendency toward a more powerful response at $64 \mathrm{~Hz}$ in the 100-day group (not shown). Looking at the frequency-response curves after the addition of atropine or TTX, we observed that atropine completely abolished the responses to EFS in six out of seven strips in the 60 -day group. One strip showed a small contraction when stimulated at $64 \mathrm{~Hz}$. However, the same strip showed small contractions at both 32 and $64 \mathrm{~Hz}$ after blocking with TTX,
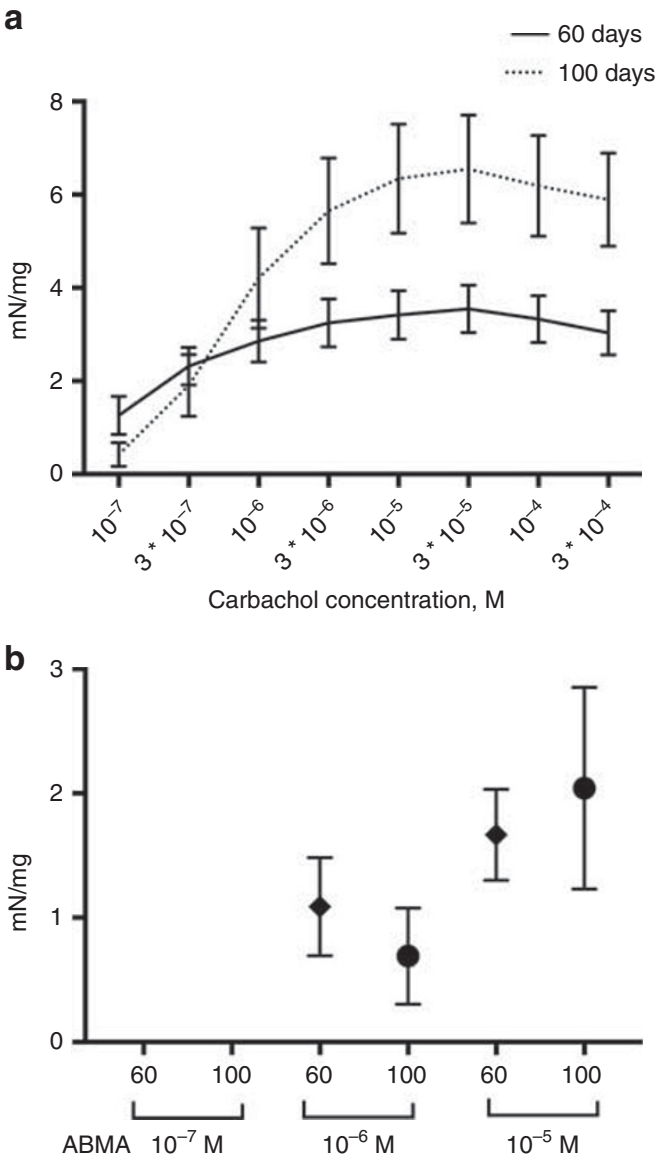

Figure 3. Pharmacological stimulation. (a) Concentration-response curves for carbachol. Carbachol was added to the organ bath chambers in cumulative doses of eight steps. At the lowest concentration, we observed a greater response in the strips from the 60-day group, whereas the overall picture was a significantly greater response in the 100-day group, where $P<0.01$. (b) Responses to stimulation with $a-\beta$ methylene-ATP (ABMA). Single doses of ABMA were added with a careful washout in-between. No strips responded to the lowest dose of $10^{-7} \mathrm{M}$ ABMA, but all strips responded to the higher doses. There was no significant difference between the groups in the mean weightadjusted responses. The error bars indicate $95 \% \mathrm{Cl}$.

indicating direct muscle stimulation (Figure 4a). In the 100day group, two strips displayed small contractions elicited by EFS after the addition of atropine, abolished by TTX in one strip (Figure $4 \mathbf{b}$ ). When adding ABMA to the preparations during the atropine blockade, we observed an initial contraction in all strips, as expected. The frequencyresponse curves measured hereafter, showed relaxation in five of the six strips in the 100-day group. This phenomenon was not observed in the 60-day group, except maybe at a high-frequency stimulation in one strip (Figure $4 \mathrm{c}-\mathrm{e}$ ).

\section{DISCUSSION}

In this study, we demonstrated that spontaneous bladder contractions were less coordinated later in gestation when the bladder gains its reservoir function. We further demonstrated a marked reduction in both the frequency and the amplitude 
of these contractions. There was an increase in contractility elicited by carbachol stimulation. Functioning purinergic pathways could be demonstrated already halfway through gestation with no significant difference in response when
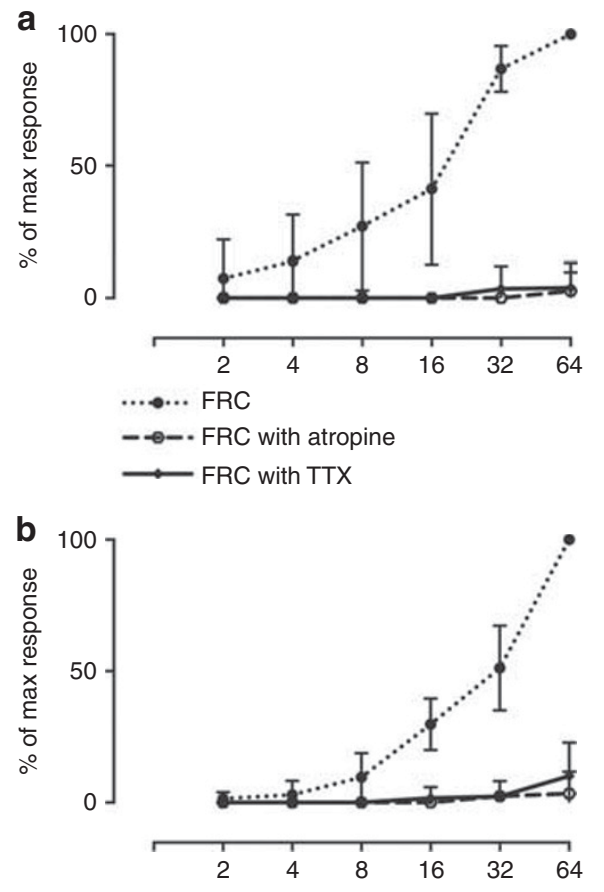

C

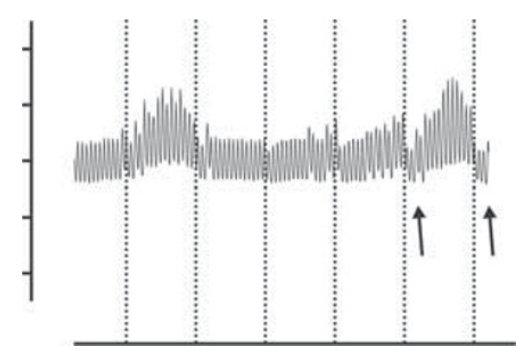

d

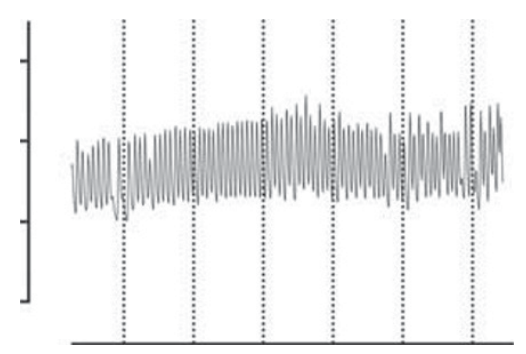

e

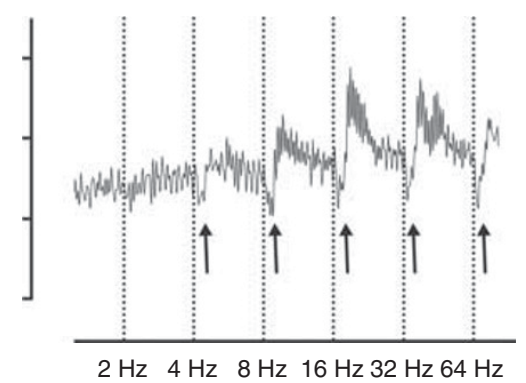

compared to the older group. However, since atropine almost entirely abolished the responses elicited by EFS, it seems that purinergic pathways do not significantly contribute to neurogenic contractions in the fetal porcine bladder. While EFS caused similar magnitudes of contractile responses in both groups, EFS during the blockade of muscarinic and purinergic receptors caused relaxations only in the 100-day group. Histologically, we demonstrated an increased ratio of the smooth muscle to other tissues in the bladder wall at 100 days, whereas the structural organization seemed to be already well established at 60 days of gestation.

Spontaneous activity of isolated bladder strips as well as whole bladders from different mammalian species has been extensively studied in adult bladders (13-17). Spontaneous activity is unaffected by the addition of atropine and TTX, and it is generally accepted that spontaneous activity is myogenic and not elicited via neural pathways. The observations on spontaneous contractile activity in human fetal bladder are scarce. Mitolo-Chieppa et al. (18) found that in human fetal bladder strips below 4 months, the spontaneous motility was "very marked", but was almost lacking in preparations of bladder strips at 6 months. Sibley (13) compared bladder strips from adult human, pig, and rabbit bladders, and found similar results from pigs and human subjects, with spontaneous activity in $71-89 \%$ of strips from the trigone region, but only in $18-20 \%$ of strips from the bladder dome. In contrast, all strips from the rabbit bladder, regardless of the place of origin, displayed a spontaneous activity. Brading (16) reviewed the information on spontaneous bladder activity in various species, the coupling between myocytes and the role of ion channels. She concluded that preparations from overactive bladders displayed an increased spontaneous activity, pointing toward a more extensive coupling between cells in these bladders. In our study, where strips were prepared from the bladder body, all preparations exhibited spontaneous contractions. A regular pattern of spontaneous activity was found at 60 days compared to a more irregular pattern at 100 days. This change may reflect a more extensive coupling between the muscle cells early during gestation, and a more "syncytial"

Figure 4. Effects of electrical field stimulation. (a, b) Overall responses to EFS normalized to the strip weight and expressed as a percentage of the maximum response in a frequency-response curve (FRC). No significant difference between the groups. (a) In the 100-day group, two strips showed small responses after blocking by atropine. One of these could be attributed to direct muscle stimulation, as there was a response remaining after the addition of $\Pi T X$. (b) In the 60-day group, one strip showed a small response after blocking by atropine, which was remaining after the addition of TTX. (c-e) Tracings from EFS in the presence of atropine and ABMA. (c) In the 60-day group, there was a possible relaxation elicited by 32 and $64 \mathrm{~Hz}$ stimulation in one strip (indicated by arrows). (d) The other six strips in the 60-day group showed no responses. (e) The arrows indicate relaxations elicited by EFS in a 100-day strip at $4 \mathrm{~Hz}$ stimulation and upwards. Five of six 100-day preparations elicited relaxations from $8 \mathrm{~Hz}$ and upwards. 
organization of the detrusor muscle. Drake et al. (15) suggested that the bladder is organized in modules of the smooth muscle, each with its own spontaneous activity. The increasing irregularity of the spontaneous contractions found at 100 days in our study may reflect the development of such modules, in parallel with the development of the striated muscle sphincter, and a change of bladder function from being a conduit to the emergence of a reservoir function and voiding ability. The observed contraction patterns were in agreement with findings in pressure-flow studies on fetal pigs. The midterm fetuses demonstrate a constant dribbling of urine and regular fluctuations in detrusor pressure, whereas fetuses later in gestation demonstrate staccato voidings inbetween periods of urine storage with irregular patterns of detrusor activity $(2,7)$.

The role of spontaneous myogenic activity and its underlying mechanisms remains unclear (16). Dean et al. (19) suggested that a myogenic tone significantly contributes to the increased stiffness observed in young fetal bladders, and Longhurst (20) speculated that a high basal tone may be a protective mechanism to keep the developing bladder empty during the early stages of gestation, before it is able to respond to distension and void actively. When examining spontaneous activity tracings by FFT, we demonstrated a higher frequency of contractions in the 60-day group, as compared to the 100day group, possibly reflecting a higher myogenic tone in the bladder. The observed increase in frequency after $\mathrm{KCl}$ activation in the 60-day group might reflect an incomplete repolarization of the muscle cells. The amplitude of spontaneous contractions was also significantly greater in the 60-day group, a finding that may reflect an inhibiting modulation that develops during the second half of gestation. This would agree with the findings of Mitolo-Chieppa et al. (18) in the human fetal bladder. In future studies, it would be interesting to further study this activity also on a cellular level, for instance considering calcium fluxes.

We found that a neurally mediated inhibitory mechanism is developed in the 100-day group, but not in the 60-day group. This is based on the demonstrated relaxations caused by EFS in the 100-day group in the presence of both atropine and ABMA. Thus, the mechanism may be mediated through noncholinergic, non-purinergic pathways. Similar relaxations induced by EFS have been demonstrated in studies on fetal lamb and calf, where neuronal release of nitric oxide (NO) was concluded to be mediating the relaxant responses $(21,22)$. Relaxations induced by EFS were demonstrated decades ago by Klarskov (23), studying adult pig and human detrusor and later confirmed by others, as reviewed by Andersson and Persson (24). Several researchers agree that NO is a likely inhibitor causing these responses, although other substances such as vasoactive intestinal polypeptide, neuropeptide $\mathrm{Y}$, and catecholamines, cannot be ruled out as possible contributors (23-27). Despite the increasing attention to these inhibitory pathways, their physiological importance remains to be uncertain. It seems reasonable to assume that the major role of relaxations is during the bladder-filling phase, to avoid a built-up of pressure as increasing volumes of urine are stored. Our results, pointing toward the development of the relaxation pathway after the development of the urethral sphincter, agree with this theory. The need for accommodation does not arise until the bladder obtains a reservoir function.

In the normal adult bladder, voiding is primarily initiated upon the release of acetylcholine and ATP from the parasympathetic nerves (28). The results from animal and human studies show diverging results with regard to the importance of the different pathways to contraction. However, most studies agree that, in the adult healthy human bladder, acetylcholine acting on the M3 receptor is by far the major cause of contraction, with only a minor, atropineresistant, contribution from ATP acting on P2X1. Adrenoceptors are also expressed in the detrusor, particularly the $\beta 3$ adrenoceptors, but play a minor role, compared to the muscarinic and purinoceptors, playing opposite roles and causing detrusor relaxation when stimulated. Several other factors are involved as modulators of the micturition reflex, but these effects are less described (28). In the present study, we demonstrated that the contractile responses to carbachol increased during gestation. Only few strips showed any atropine resistance to a very small extent. These results differ from findings on a number of studies on bladders from adult animals, where up to $80 \%$ of atropine resistance has been described, indicating a major role of purinergic signaling in some cases (13). Studies on pathological adult human bladders such as overactive or obstructed bladders, also showed an increased atropine resistance $(29,30)$. As previous studies on adult porcine bladder have shown up to $20 \%$ of atropine resistance, and we found almost no atropine resistance in the fetal preparations, there may be a developmental change involved (13). It seems that there is a significant difference between the species concerning the developmental aspects of bladder function, and which animal model best reflects what is happening in humans remains a question. There is ample morphological information on the development of the human bladder (31-34). However, information on functional characteristics is scarce (18). To our knowledge, this is the first in vitro study on fetal porcine bladders. The comparison of two time points in gestation is valuable and corroborates the findings of previous urodynamic studies (2). However, it was only possible to examine a limited number of drugs, and as we obtained several fetuses from the same sow, they were genetically similar. Further studies on the functional development of the fetal bladder are desirable, and a model with translational impact is needed.

\section{CONCLUSION}

Spontaneous contractions occur both at 60 and 100 days of gestation, but are less coordinated as the bladder gains its reservoir function. Quantitatively, the contractility of the bladder increases during gestation. The responses to purinergic agonists can be demonstrated as early as halfway through gestation, but as atropine almost entirely abolished 
the responses elicited by EFS, the purinergic pathways do not seem to contribute to neurally mediated contractions. Relaxations elicited by EFS, in the presence of muscarinic and purinergic blockade, suggest the development of an inhibitory neurogenic pathway between the midterm and full term of gestation The present data suggest that the fetal pig bladder may be a model with translational impact that can be useful for further studies on the development of bladder function.

\section{ACKNOWLEDGMENTS}

We highly appreciate the technical assistance from the laboratory technician Kristina Lystlund Lauridsen in the processing of histological examinations, and the assistance from Simon Gabriel Comerma Steffensen and Yutao Lu during the in vitro studies. Performing and interpreting the FFT would not have been possible without the kind help from the engineer, Martin Nors Skov, and a professor in physics, Michael Pedersen.

\section{STATEMENT OF FINANCIAL SUPPORT}

This study was supported by a grant from the Lundbeck Foundation.

Disclosure: The authors declare no conflict of interest.

\section{REFERENCES}

1. Cuckow PM Embryology of the urogenital tract. In: Gearhart JP, Rink RC, Mouriquand PDE, eds. Pediatric Urology. US: Saunders, 2010. pp. 1-10.

2. Olsen LH, Dalmose AL, Swindle MM, Jørgensen TM, Djurhuus JC. Male fetal pig lower urinary tract function in mid second and early third trimester of gestation. J Urol 2001;165:2331-4.

3. Ludwikowski B, Oesch Hayward I, Brenner E, Fritsch H. The development of the external urethral sphincter in humans. BJU Int 2001;87:565-8.

4. Tasian G, Cunha G, Baskin L. Smooth muscle differentiation and patterning in the urinary bladder. Differentiation 2010;80:106-17.

5. Nyirady P, Thiruchelvam N, Godley ML, David A, Cuckow PM, Fry CH. Contractile properties of the developing fetal sheep bladder. Neurourol Urodyn 2005;24:276-81.

6. Lee JG, Macarak E, Coplen D, Wein AJ, Levin RM. Distribution and function of the adrenergic and cholinergic receptors in the fetal calf bladder during mid-gestational age. Neurourol Urodyn 1993;12:599-607.

7. Olsen LH, Dalmose AL, Swindle MM, Djurhuus JC, Jørgensen TM. Male fetal pig lower urinary tract function. Part II: free voiding pattern close to term and in the newborn. J Urol 2004;171:2660-3.

8. Gilpin SA, Gosling JA. Smooth muscle in the wall of the developing human urinary bladder and urethra. J Anat 1983;137 (Pt 3): 503-12.

9. Crowe R, Burnstock G. A histochemical and immunohistochemical study of the autonomic innervation of the lower urinary tract of the female pig. Is the pig a good model for the human bladder and urethra? J Urol 1989;141:414-22.

10. Guan Z, Kiruluta G, Coolsaet B, Elhilali M. Conscious minipig model for evaluating the lower urinary tract. Neurourol Urodyn 1994;13:147-58.

11. Dalmose AL, Hvistendahl JJ, Olsen LH, Eskild-Jensen A, Djurhuus JC, Swindle MM. Surgically induced urologic models in swine. J Invest Surg 2000;13:133-45.

12. National Research Council (US) Committee for the Update of the Guide for the Care and Use of Laboratory Animals Guide for the Care and Use of Laboratory Animals. Washington, DC: National Academies Press, 2011.
13. Sibley GN. A comparison of spontaneous and nerve-mediated activity in bladder muscle from man, pig and rabbit. J Physiol 1984;354:431-43.

14. Van Duyl WA. Spontaneous contractions in urinary bladder smooth muscle: preliminary results. Neurourol Urodyn 1985;4:301-7.

15. Drake MJ, Harvey IJ, Gillespie JI. Autonomous activity in the isolated guinea pig bladder. Exp Physiol 2003;88:19-30.

16. Brading AF. Spontaneous activity of lower urinary tract smooth muscles: correlation between ion channels and tissue function. J Physiol 2005;570: 13-22.

17. Drake MJ, Kanai A, Bijos DA, et al. The potential role of unregulated autonomous bladder micromotions in urinary storage and voiding dysfunction; overactive bladder and detrusor underactivity. BJU International 2017;119:22-9.

18. Mitolo-Chieppa D, Schönauer S, Grasso G, Cicinelli E, Carratú MR. Ontogenesis of autonomic receptors in detrusor muscle and bladder sphincter of human fetus. Urology 1983;21:599-603.

19. Dean GE, Cargill RS, Macarak E, Snyder HM, Duckett JW, Levin R. Active and passive compliance of the fetal bovine bladder. J Urol 1997;158:1094-9.

20. Longhurst P. Developmental aspects of bladder function. Scand J Urol Nephrol Suppl 2004;38:11-9.

21. Levin RM, Macarak E, Howard P, Horan P, Kogan BA. The response of fetal sheep bladder tissue to partial outlet obstruction. J Urol 2001;166: 1156-60.

22. Lee JG, Coplen D, Macarak E, Wein AJ, Levin RM. Comparative studies on the ontogeny and autonomic responses of the fetal calf bladder at different stages of development: involvement of nitric oxide on field stimulated relaxation. J Urol 1994;151:1096-101.

23. Klarskov P. Non-cholinergic, non-adrenergic nerve-mediated relaxation of pig and human detrusor muscle in vitro. Br J Urol 1987;59:414-9.

24. Andersson KE, Persson K. Nitric oxide synthase and nitric oxidemediated effects in lower urinary tract smooth muscles. World J Urol 1994;12:274-80.

25. Hills J, Meldrum LA, Klarskov P, Burnstock G. A novel non-adrenergic, non-cholinergic nerve-mediated relaxation of the pig bladder neck: an examination of possible neurotransmitter candidates. Eur J Pharmacol 1984;99:287-93.

26. James MJ, Birmingham AT, Hill SJ. Partial mediation by nitric oxide of the relaxation of human isolated detrusor strips in response to electrical field stimulation. Br J Clin Pharmacol 1993;35:366-72.

27. Jen PY, Dixon JS, Gosling JA. Immunohistochemical localization of neuromarkers and neuropeptides in human fetal and neonatal urinary bladder. Br J Urol 1995;75:230-5.

28. Andersson K-E, Arner A. Urinary bladder contraction and relaxation: physiology and pathophysiology. Physiol Rev 2004;84:935-86.

29. Bayliss M, Wu C, Newgreen D, Mundy AR, Fry CH. A quantitative study of atropine-resistant contractile responses in human detrusor smooth muscle, from stable, unstable and obstructed bladders. J Urol 1999;162: 1833-9.

30. Andersson K-E, Hedlund P. Pharmacologic perspective on the physiology of the lower urinary tract. Urology 2002;60:13-20 discussion20-1.

31. Kim KM, Kogan BA, Massad CA, Huang YC. Collagen and elastin in the normal fetal bladder. J Urol 1991;146:524-7.

32. Freedman AL, Qureshi F, Shapiro E, et al. Smooth muscle development in the obstructed fetal bladder. Urology 1997;49:104-7.

33. Sulak O, Cankara N, Malas MA, Koyuncu E, Desdicioglu K. Anatomical development of urinary bladder during the fetal period. Clin Anat 2008;21:683-90.

34. Favorito LA, Pazos HM, Costa SF, Costa WS, Sampaio FJ. Morphology of the fetal bladder during the second trimester: comparing genders. J Pediatr Urol 2014;10:1014-9. 